\title{
A Remark on Asymptotic Completeness of Local Fields
}

\author{
Max Rinke \\ Seminar für theoretische Physik der ETH Zürich \\ Received February 18, 1969
}

\begin{abstract}
Assuming the existence of an asymptotically complete Wightman field with non-trivial $S$-matrix, we construct a local field such that the Haag-Ruelle scattering theory applied to this field leads to $\mathfrak{S}_{\text {in }} \neq \mathfrak{S}$ and $\mathfrak{S}_{\text {in }} \neq \mathfrak{S}_{\text {out }}$.
\end{abstract}

In the framework of local field theory one can define, using the HAAG-RUELLE [1] scattering theory, incoming and outgoing states and the corresponding Hilbert spaces $\mathfrak{S}_{\text {in }}$ and $\mathfrak{S}_{\text {out }}$. It is well-known that the axiom of asymptotic completeness $\left(\mathfrak{S}_{\text {in }}=\mathfrak{S}\right)$ is independent of the other axioms of field theory. In order to have an unitary $S$-matrix, it is sufficient to require $\mathfrak{S}_{\text {in }}=\mathfrak{S}_{\text {out }}$. Starting from an asymptotically complete Wightman field with non-trivial $S$-matrix we shall construct a field which does not fulfill this requirement. The construction will show that in our case asymptotic completeness and unitarity of the $S$-matrix are destroyed by the fact that the functional of truncated vacuum expectation values can be decomposed into a sum of two such (truncated) functionals.

In the following we consider real scalar Wightman fields. We denote the field operator by $A(x)$, the vacuum state by $\Omega$, the representation of the inhomogeneous Lorentz group by $U(a, \Lambda)$ and the Hilbert space by $\mathfrak{S}$.

In addition to the usual postulates of field theory we require [2]:

(I) Let $\sigma(P)$ be the spectrum of the energy momentum operator $P$. Then $\sigma(P)$ has the form:

$\sigma(P)=\{p \mid p=0\} \cup\left\{p \mid p_{0}>0, p^{2}=m^{2}\right\} \cup\left\{p \mid p_{0}>0, p^{2} \geqq 4 m^{2}\right\} ; m>0$.

(II) Let $\mathfrak{G}_{1}$ be defined by $\mathfrak{G}_{1}=\left\{\Phi \mid \Phi \in \mathfrak{Y},\left(P^{2}-m^{2}\right) \Phi=0\right\}$, and let $U_{1}(a, \Lambda)$ be the representation of the inhomogeneous Lorentz group in $\mathfrak{G}_{1}$. Then $U_{1}(a, \Lambda)$ is an irreducible representation and has spin 0 .

(III) Let $P_{1}$ be the projection on $\mathfrak{G}_{1}$. Then the following is true:

$$
\left(A(x) \Omega, P_{1} A(y) \Omega\right)=i \Delta^{(+)}\left(m^{2}, x-y\right) .
$$

With the notation (taken from a paper by HEPP [3])

$$
G=\left\{p\left|p_{0}<0,\right| p^{2}-m^{2} \mid<\frac{m^{2}}{2}\right\}
$$


and

we define

$$
S(G)=\left\{g \mid g \in S\left(R^{4}\right), \operatorname{supp} g \subset G\right\}
$$

$$
A(f, t)=\int \widetilde{A}(p) \tilde{f}(p) e^{-i\left(p_{0}+\omega\right) t} d^{4} p ; \omega=\sqrt{m^{2}+|\boldsymbol{p}|^{2}}, \tilde{f} \in S(G) .
$$

HAAG and RUELLE [1] have shown that the strong limits

$$
\lim _{t \rightarrow \mp \infty} \prod_{j=1}^{n} A\left(f_{j}, t\right) \Omega=\Phi_{\text {in }}\left(f_{1}, \ldots f_{n}\right)
$$

exist and define incoming and outgoing states. The Hilbert space spanned by $\Omega$ and the states $\Phi_{\text {in }}$ is denoted by $\mathfrak{S}_{\text {in }}$. Asymptotic completeness of the field $A$ means: $\mathfrak{S}_{\text {in }}=\mathfrak{Y}$.

If we have two fields $A_{1}(x), A_{2}(x)$ with the vacuum states $\Omega_{1}, \Omega_{2}$ and with the representations $U_{1}(a, \Lambda), U_{2}(a, \Lambda)$ of the inhomogeneous Lorentz group, we can construct a new field $B(x)$ by

$$
\begin{aligned}
B(x) & =A_{1}(x) \otimes 1+1 \otimes A_{2}(x) ; \\
\Omega & =\Omega_{1} \otimes \Omega_{2} ; \\
U(a, \Lambda) & =\left[U_{1}(a, \Lambda) \otimes U_{2}(a, \Lambda)\right]_{B} .
\end{aligned}
$$

$\left[U_{1}(a, \Lambda) \otimes U_{2}(a, \Lambda)\right]_{B}$ is the restriction of $U_{1}(a, \Lambda) \otimes U_{2}(a, \Lambda)$ to the space $\mathfrak{S}^{(B)}=\overline{\mathfrak{A}_{B} \Omega} \leqq \overline{\mathfrak{F}^{\left(A_{1}\right)} \otimes \mathfrak{S}^{\left(A_{2}\right)}}$. $\left(\mathfrak{A}_{B}\right.$ is the polynomial algebra of $\left.B\right)$.

This construction was introduced by Borchers [4]. From (2) we obtain for the truncated vacuum expectation values (TVEV):

$$
\begin{aligned}
& \left(\Omega, B\left(x_{1}\right) \ldots B\left(x_{n}\right) \Omega\right)^{T} \\
& \quad=\left(\Omega_{1}, A_{1}\left(x_{1}\right) \ldots A_{1}\left(x_{n}\right) \Omega_{1}\right)^{T}+\left(\Omega_{2}, A_{2}\left(x_{1}\right) \ldots A_{2}\left(x_{n}\right) \Omega_{2}\right)^{T} .
\end{aligned}
$$

(2) and (3) are equivalent statements, and we shall use both of them. We are now prepared for the following

Theorem. Let $\left\{A(x), \Omega_{A}, U_{A}(a, \Lambda)\right\}$ be a local field theory which satisfies the conditions (I), (II), (III). Let $A$ be asymptotically complete, and let $S_{A}$ be the corresponding $S$-matrix. Then the field theory defined by

$$
\begin{gathered}
B(x)=\frac{1}{\sqrt{2}}(A(x) \otimes 1+1 \otimes A(x)) ; \quad \Omega_{B}=\Omega_{A} \otimes \Omega_{A} \\
U_{B}(a, \Lambda)=\left[U_{A}(a, \Lambda) \otimes U_{A}(a, \Lambda)\right]_{B}
\end{gathered}
$$

has the following properties:

1. The theory (4) satisfies the conditions (I), (II), (III).

2. $\mathfrak{H}_{\text {in }}^{(B)}=\mathfrak{S}^{(B)}$ if and only if $A$ is a free field.

3. $\mathfrak{H}_{\text {in }}^{(B)}=\mathfrak{H}_{\text {out }}^{(B)}$ if and only if $S_{A}=1$.

Proof. 1. The requirements (I), (III) are fulfilled by construction. Hence we have only to show that (II) is fulfilled. 
We define

$$
\begin{aligned}
& \mathfrak{H}_{1}^{(B)}=\left\{\Phi \mid \Phi \in \mathfrak{G}^{(B)},\left(P^{2}-m^{2}\right) \Phi=0\right\} ; \\
& \hat{\mathfrak{G}}_{1}^{(B)}=\overline{\left\{B(f) \Omega_{B} ; \hat{f} \in S(G)\right\}} .
\end{aligned}
$$

The representation of the inhomogeneous Lorentz group in $\hat{\mathfrak{G}}_{1}^{(B)}$ is irreducible and has spin 0 . We want to show: $\mathfrak{G}_{1}^{(B)}=\hat{\mathfrak{G}}_{1}^{(B)}$.

Let us consider $\mathfrak{S}^{(B)}$ as a subspace of $\mathfrak{G}^{\prime}=\overline{\mathfrak{S}^{(A)} \otimes \mathfrak{S}^{(A)}}$ with $U_{A}(a, \Lambda) \otimes U_{A}(a, \Lambda)$ as the representation of the inhomogeneous Lorentz group. We define:

$$
\mathfrak{H}_{1}^{\prime}=\left\{\Phi \mid \Phi \in \mathfrak{S}^{\prime},\left(P^{2}-m^{2}\right) \Phi=0\right\}
$$

Since $A$ satisfies condition (II), we get

$$
\begin{aligned}
\mathfrak{S}_{1}^{\prime} & =\mathfrak{S}_{1}^{(A)} \otimes \Omega_{A} \oplus \Omega_{A} \otimes \mathfrak{S}_{1}^{(A)} \\
& =\overline{\left\{A\left(f_{1}\right) \Omega_{A} \otimes \Omega_{A}+\Omega_{A} \otimes A\left(f_{2}\right) \Omega_{A} ; \tilde{f}_{1}, \tilde{f}_{2} \in S(G)\right\}} .
\end{aligned}
$$

$\hat{\mathfrak{G}}_{1}^{(B)}$ and $\mathfrak{S}_{1}^{(B)}$ are subspaces of $\mathfrak{S}_{1}^{\prime}$. Let $\hat{\mathfrak{S}}_{1}^{(B) \perp}$ be the orthogonal complement of $\widehat{\mathfrak{G}}_{1}^{(B)}$ with respect to $\mathfrak{G}_{1}^{\prime}$. Then we have:

$$
\hat{\mathfrak{G}}_{1}^{(B) \perp}=\overline{\left\{A(f) \Omega_{A} \otimes \Omega_{A}-\Omega_{A} \otimes A(f) \Omega_{A} ; \tilde{f} \in \overline{S(G)}\right\}} .
$$

Let us now consider the scalar products:

$$
\begin{gathered}
\left(A(f) \Omega_{A} \otimes \Omega_{A}-\Omega_{A} \otimes A(f) \Omega_{A},\left[\prod_{j=1}^{n} B\left(g_{j}\right)\right] \Omega_{A} \otimes \Omega_{A}\right) ; \\
n=1,2, \ldots ; \quad f, g_{j} \in S\left(R^{4}\right) .
\end{gathered}
$$

Since $B$ is symmetric in $A \otimes 1$ and $1 \otimes A$, we get:

$$
\begin{aligned}
& \left([A(f) \otimes 1] \Omega_{A} \otimes \Omega_{A},\left[\prod_{j=1}^{n} B\left(g_{\jmath}\right)\right] \Omega_{A} \otimes \Omega_{A}\right) \\
= & \left([1 \otimes A(f)] \Omega_{A} \otimes \Omega_{A},\left[\prod_{j=1}^{n} B\left(g_{j}\right)\right] \Omega_{A} \otimes \Omega_{A}\right) .
\end{aligned}
$$

From this we conclude that the scalar products (5) vanish for arbitrary $f, g_{j} \in S\left(R^{4}\right)$. Therefore $\hat{\mathfrak{H}}_{1}^{(B) \perp}$ is orthogonal to $\mathfrak{G}^{(B)}$. This implies $\hat{\mathfrak{H}}_{1}^{(B)}=\mathfrak{H}_{1}^{(B)}$.

Hence $B$ satisfies condition (II).

2. If $A$ is a free field, $B$ is also a free field and, of course, asymptotically complete. It remains to show that asymptotic completeness of $B$ implies that $A$ is a free field. 
We now suppose that $B$ is asymptotically complete. With the operators $A\left(f_{j}, t\right), B\left(f_{j}, t\right)$ given by (1) we construct the states

$$
\begin{aligned}
& \Phi_{\mathrm{in}}^{(B)}\left(f_{1}, \ldots f_{n}\right)=\lim _{t \rightarrow-\infty} \prod_{j=1}^{n} B\left(f_{j}, t\right) \Omega_{B} ; \\
& \Phi_{\mathrm{in}}^{(A)}\left(f_{1}, \ldots f_{n}\right)=\lim _{t \rightarrow-\infty} \prod_{j=1}^{n} A\left(f_{j}, t\right) \Omega_{A} .
\end{aligned}
$$

The linear hull of all such states is called $D_{n \mid \text { in }}^{(B)}$ resp. $D_{n \mid \text { in }}^{(A)}$. With $D_{0 \mid \text { in }}^{(B)}=\left\{\lambda \Omega_{B}\right\}, D_{0 \mid \text { in }}^{(A)}=\left\{\lambda \Omega_{A}\right\}$ we define

$$
D_{\mathrm{in}}^{(B)}=\bigoplus_{n=0}^{\infty} D_{n \mid \mathrm{in}}^{(B)} ; D_{\mathrm{in}}^{(A)}=\bigoplus_{n=0}^{\infty} D_{n \mid \mathrm{in}}^{(A)} .
$$

$D_{\mathrm{in}}^{(B)}\left(\operatorname{resp} . D_{\mathrm{in}}^{(A)}\right)$ is dense in $\mathfrak{G}_{\mathrm{in}}^{(B)}\left(\operatorname{resp} . \mathfrak{G}_{\mathrm{in}}^{(A)}\right)$. Finally we remark that the mapping $\Phi_{\text {in }}^{(B)}\left(f_{1}, \ldots f_{n}\right) \rightarrow \Phi_{\text {in }}^{(A)}\left(f_{1}, \ldots f_{n}\right)$ can be extended to an isometric mapping of $\mathfrak{G}_{\mathrm{in}}^{(B)}$ onto $\mathfrak{G}_{\mathrm{in}}^{(A)}$. With

$$
j_{B}(x)=\left(\square+m^{2}\right) B(x) \quad \text { and } \quad j_{A}(x)=\left(\square+m^{2}\right) A(x)
$$

we get:

$\lim _{t \rightarrow-\infty}\left(j_{B}(g) \Omega_{B}, \prod_{j=1}^{n} B\left(f_{j}, t\right) \Omega_{B}\right)=\lim _{t \rightarrow-\infty}\left(j_{B}(g) \Omega_{B}, \prod_{j=1}^{n} B\left(f_{j}, t\right) \Omega_{B}\right)^{T}$, $\lim _{t \rightarrow-\infty}\left(j_{A}(g) \Omega_{A}, \prod_{j=1}^{n} A\left(f_{j}, t\right) \Omega_{A}\right)=\lim _{t \rightarrow-\infty}\left(j_{A}(g) \Omega_{A}, \prod_{j=1}^{n} A\left(f_{j}, t\right) \Omega_{A}\right)^{T^{\prime}}$,

Due to (3), we obtain

$$
g \in S\left(R^{4}\right) \text {. }
$$

and

$$
\left(\Omega_{B}, B\left(x_{1}\right) \ldots B\left(x_{n}\right) \Omega_{B}\right)^{T}=\frac{2}{\sqrt{2^{n}}}\left(\Omega_{A}, A\left(x_{1}\right) \ldots A\left(x_{n}\right) \Omega_{A}\right)^{T}
$$

$$
\left(j_{B}(g) \Omega_{B}, \Phi_{\mathrm{in}}^{(B)}\left(f_{1}, \ldots f_{n}\right)\right)=\frac{2}{\sqrt{2}^{n+1}}\left(j_{A}(g) \Omega_{A}, \Phi_{\mathrm{in}}^{(A)}\left(f_{1}, \ldots f_{n}\right)\right) .
$$

Let $\Phi_{\mathrm{in}}^{(B)}=\sum_{n} \Phi_{n \mid \mathrm{in}}^{(B)}, \Phi_{n \mid \mathrm{in}}^{(B)} \in D_{n \mid \mathrm{in}}^{(B)}$, and let $\Phi_{\mathrm{in}}^{(A)}$ be the corresponding state in $D_{\mathrm{in}}^{(A)}$. Then we get:

$$
\sum_{n}\left(j_{B}(g) \Omega_{B}, \Phi_{n \mid \text { in }}^{(B)}\right)=\sum_{n} \frac{2}{\sqrt{2^{n+1}}}\left(j_{A}(g) \Omega_{A}, \Phi_{n \mid \mathrm{in}}^{(A)}\right) .
$$

Since $j_{B}(g) \Omega_{B} \perp \Omega_{B} \oplus \mathfrak{G}_{1}^{(B)}$ and $j_{A}(g) \Omega_{A} \perp \Omega_{A} \oplus \mathfrak{H}_{1}^{(A)}$, only terms with $n \geqq 2$ contribute to the sums. This leads to the following estimate for the right hand side of (6):

$$
\left|\left(j_{A}(g) \Omega_{A}, \sum_{n \geqq 2} \frac{1}{\sqrt{2^{n-1}}} \Phi_{n \mid \mathrm{in}}^{(A)}\right)\right| \leqq\left\|j_{A}(g) \Omega_{A}\right\| \frac{1}{\sqrt{2}}\left\|\sum_{n} \Phi_{n \mid \mathrm{in}}^{(A)}\right\| .
$$


Since $\left\|\Phi_{\text {in }}^{(B)}\right\|=\left\|\Phi_{\text {in }}^{(A)}\right\|$, we have

$$
\frac{\left|\left(j_{B}(g) \Omega_{B}, \Phi_{\text {in }}^{(B)}\right)\right|}{\left\|\Phi_{\text {in }}^{(B)}\right\|} \leqq \frac{1}{\sqrt{2}}\left\|j_{A}(g) \Omega_{A}\right\|
$$

$D_{\text {in }}^{(B)}$ is dense in $\mathfrak{H}_{\mathrm{in}}^{(B)}$, and $B$ is supposed to be asymptotically complete. We conclude:

$$
\left\|j_{B}(g) \Omega_{B}\right\| \leqq \frac{1}{\sqrt{2}}\left\|j_{A}(g) \Omega_{A}\right\|
$$

Due to (3), the 2-point functions of $A$ and $B$ are the same. Therefore we get

$$
\left\|j_{A}(g) \Omega_{A}\right\| \leqq \frac{1}{\sqrt{2}}\left\|j_{A}(g) \Omega_{A}\right\|
$$

This implies $j_{A}(g) \Omega_{A}=0$. The conclusion holds for arbitrary $g \in S\left(R^{4}\right)$. Since $A$ is local, $A$ is a free field.

3. From $S_{A}=1$ it follows:

$$
\begin{aligned}
& \lim _{t \rightarrow \infty}\left(\prod_{j=1}^{n_{1}} B\left(f_{j}, t\right) \Omega_{B}, \prod_{k=1}^{n_{2}} B\left(g_{k},-t\right) \Omega_{B}\right)^{T} \\
&= \lim _{t \rightarrow \infty} \frac{2}{{\sqrt{2^{n_{1}+n_{2}}}}^{T}}\left(\prod_{j=1}^{n_{1}} A\left(f_{j}, t\right) \Omega_{A}, \prod_{k=1}^{n_{3}} A\left(g_{k},-t\right) \Omega_{A}\right)^{T^{\top}}=0, \\
& n_{1}+n_{2}>2 .
\end{aligned}
$$

This yields

$$
\lim _{t \rightarrow+\infty} \prod_{j=1}^{n} B\left(f_{j}, t\right) \Omega_{B}=\lim _{t \rightarrow-\infty} \prod_{j=1}^{n} B\left(f_{j}, t\right) \Omega_{B} .
$$

Hence we have $\mathfrak{G}_{\text {in }}^{(B)}=\mathfrak{H}_{\text {out }}^{(B)}$. It remains to show that the assumption $\mathfrak{H}_{\text {in }}^{(B)}=\mathfrak{H}_{\text {out }}^{(B)}$ implies $S_{A}=1$.

We now assume $\mathfrak{G}_{\mathrm{in}}^{(B)}=\mathfrak{S}_{\text {out }}^{(B)}$. We want to give a proof by induction. We define:

$$
\begin{aligned}
& \Psi^{(A)}\left(f_{1}, \ldots f_{n}\right)=\Phi_{\text {out }}^{(A)}\left(f_{1}, \ldots f_{n}\right)-\Phi_{\text {in }}^{(A)}\left(f_{1}, \ldots f_{n}\right), \\
& \Psi^{(B)}\left(f_{1}, \ldots f_{n}\right)=\Phi_{\text {out }}^{(B)}\left(f_{1}, \ldots f_{n}\right)-\Phi_{\text {in }}^{(B)}\left(f_{1}, \ldots f_{n}\right) .
\end{aligned}
$$

For $n=1$ we have $\Psi^{(A)}(f)=0$. Suppose now, it has been proved that $\Psi^{(A)}\left(f_{1}, \ldots f_{n}\right)$ vanishes for all $n<N$ and arbitrary $f_{j}, \tilde{f}_{j} \in S(G)$, $j=1,2 \ldots n$. Since the TVEV of $B$ are multiples of the TVEV of $A$, $\Psi{ }^{(B)}\left(f_{1}, \ldots f_{n}\right)$ also vanishes for $n<N$. This has the consequence:

$$
\begin{aligned}
& \left(\Psi^{(B)}\left(f_{1}, \ldots f_{N}\right), \Phi_{\mathrm{in}}^{(B)}\left(g_{1}, \ldots g_{l}\right)\right) \\
& =\lim _{t \rightarrow \infty}\left(\left\{\prod_{j=1}^{N} B\left(f_{j}, t\right)-\prod_{j=1}^{N} B\left(f_{j},-t\right)\right\} \Omega_{B}, \prod_{k=1}^{l} B\left(g_{k},-t\right) \Omega_{B}\right)^{T^{\prime}} \\
& =\lim _{t \rightarrow \infty} \frac{2}{\sqrt{2}^{N+l}}\left(\left\{\prod_{j=1}^{N} A\left(f_{j}, t\right)-\prod_{j=1}^{N} A\left(f_{j},-t\right)\right\} \Omega_{A}, \prod_{k=1}^{l} A\left(g_{k},-t\right) \Omega_{A}\right)^{T} \\
& =\frac{2}{\sqrt{2^{N+l}}}\left(\Psi^{(A)}\left(f_{1}, \ldots f_{N}\right), \Phi_{\mathrm{in}}^{(A)}\left(g_{1}, \ldots g_{l}\right)\right) .
\end{aligned}
$$


Let $\Phi_{\mathrm{in}}^{(B)}, \Phi_{\mathrm{in}}^{(A)}$ be the states which we used in the proof of statement 2$)$. Then we get:

$$
\left(\Psi^{(B)}\left(f_{1}, \ldots f_{N}\right), \sum_{n} \Phi_{n \mid \mathrm{in}}^{(B)}\right)=\left(\Psi^{(A)}\left(f_{1}, \ldots f_{N}\right), \sum_{n} \frac{2}{\sqrt{2^{N+n}}} \Phi_{n \mid \mathrm{in}}^{(A)}\right) .
$$

Due to the induction assumption, only terms with $n \geqq N$ contribute to the sums.

This leads to the following estimates:

$$
\begin{gathered}
\left|\left(\Psi^{(A)}\left(f_{1}, \ldots f_{N}\right), \sum_{n \geqq N} \Phi_{n \mid \text { in }}^{(A)} \frac{2}{\sqrt{2^{N+n}}}\right)\right| \leqq\left\|\Psi^{(A)}\left(f_{1}, \ldots f_{N}\right)\right\| \frac{1}{2^{N-1}}\left\|\sum_{n} \Phi_{n \mid \text { in }}^{(A)}\right\| \\
\frac{\left|\left(\Psi^{(B)}\left(f_{1}, \ldots f_{N}\right), \Phi_{\text {in }}^{(B)}\right)\right|}{\left\|\Phi_{\text {in }}^{(B)}\right\|} \leqq \frac{1}{2^{N-1}}\left\|\Psi^{(A)}\left(f_{1}, \ldots f_{N}\right)\right\| .
\end{gathered}
$$

Since $\mathfrak{G}_{\text {in }}^{(B)}=\mathfrak{H}_{\text {out }}^{(B)}, \Psi^{(B)}\left(f_{1}, \ldots f_{N}\right)$ is a vector in $\mathcal{G}_{\text {in }}^{(B)}$. We conclude:

$$
\left\|\Psi^{(B)}\left(f_{1}, \ldots f_{N}\right)\right\| \leqq \frac{1}{2^{N-1}}\left\|\Psi^{(A)}\left(f_{1}, \ldots f_{N}\right)\right\|
$$

On the other hand, we have

$$
\begin{aligned}
\left\|\Psi(B)\left(f_{1}, \ldots f_{N}\right)\right\|^{2}= & \lim _{t \rightarrow \infty}\left(\left\{\prod_{j=1}^{N} B\left(f_{j}, t\right)-\prod_{j=1}^{N} B\left(f_{j},-t\right)\right\} \Omega_{B},\right. \\
& \left.\cdot\left\{\prod_{j=1}^{N} B\left(f_{j}, t\right)-\prod_{j=1}^{N} B\left(f_{j},-t\right)\right\} \Omega_{B}\right)^{T^{\prime}} \\
= & \frac{1}{2^{N-1}} \lim _{\rightarrow \infty}\left(\left\{\prod_{j=1}^{N} A\left(f_{j}, t\right)-\prod_{j=1}^{N} A\left(f_{j},-t\right)\right\} \Omega_{A},\right. \\
& \left.\cdot\left\{\prod_{j=1}^{N} A\left(f_{j}, t\right)-\prod_{j=1}^{N} A\left(f_{j},-t\right)\right\} \Omega_{A}\right)^{T} \\
= & \frac{1}{2^{N-1}}\left\|\Psi(A)\left(f_{1}, \ldots f_{N}\right)\right\|^{2} .
\end{aligned}
$$

This yields

$$
\frac{1}{\sqrt{2^{N-1}}}\left\|\Psi^{(A)}\left(f_{1}, \ldots f_{N}\right)\right\| \leqq \frac{\mathrm{J}}{2^{N-1}}\left\|\Psi^{(A)}\left(f_{1}, \ldots f_{N}\right)\right\|
$$

That implies $\Psi^{(A)}\left(f_{1}, \ldots f_{N}\right)=0$. Since the induction assumption is true for $n=1$, we get for all $n \Psi^{(A)}\left(f_{1}, \ldots f_{n}\right)=0$. The conclusion holds for arbitrary $f_{j}, \tilde{f}_{j} \in S(G)$. Hence we obtain $S_{A}=1$. This proves the theorem.

Assume now, there is an asymptotically complete Wightmann field $A(x)$ which satisfies the conditions (I), (II), (III). Let the $S$-matrix be non-trivial. Then we construct the field $B(x)=\frac{1}{\sqrt{2}}(A(x) \otimes 1+1 \otimes A(x))$. Due to our theorem we get $\mathfrak{G}_{\text {in }}^{(B)} \neq \mathfrak{H}^{(B)}$ and $\mathfrak{S}_{\text {in }}^{(B)} \neq \mathfrak{H}_{\text {out }}^{(B)}$. 
The author thanks Prof. K. Hepp and Prof. R. Jost for their interest and the hospitality at the ETH Zürich. He thanks the Schweizerische Nationalfonds and the Deutsche Forschungsgemeinschaft for financial support.

\section{References}

1. Haag, R.: Phys. Rev. 112, 669 (1958). - D. Ruelle: Helv. Phys. Acta 35, 147 (1962).

2. Jost, R.: The general theory of quantized fields, p. 119. Providence: Am. Math. Soc. 1965.

3. Hepp, K.: Commun. Math. Phys. 1, 95 (1965).

4. Borchers, H. J.: (unpublished).

M. RINKE

II. Institut f. Theoret. Physik

der Universität

2000 Hamburg 50

Luruper Chaussee 149 